\title{
Electron Beam Lithography Simulation for Subquartermicron and High Density Patterns
}

\author{
Ioannis Raptis, Nikos Glezos \\ Institute of Microelectronics, NCSR "DEMOKRITOS" \\ 15310 Ag. Paraskevi Attikis, GREECE \\ raptis@imel.demokritos.gr
}

\begin{abstract}
A fast simulation tool for electron beam lithography is applied for the prediction of energy deposition and the resist profile of high resolution patterns. For the exposure part, an analytical solution based on the Boltzmann transport equation is used. The energy deposition has been combined with analytical functions describing the resist development and complete simulation of dense layouts in the sub-quarter-micron range has been carried out. The simulation results have been compared with experimental ones and found in very good agreement.
\end{abstract}

\section{Introduction}

Electron beam lithography (EBL) has been used intensively for the development of prototype devices and for the exploration of future devices with very small critical dimensions (nanotechnology). Additionally, EBL plays an important role in mask making processes for ICs with critical dimension in the sub $0.25 \mu \mathrm{m}$ range. In the future, its use is expected to increase through the application of EBL tools in mass production in mix and match lithography schemes and in nanotechnology. Therefore the understanding of electron beam interactions with matter and the physicochemical changes associated with these interactions are of vital importance for the optimization of the whole lithographic process.

Lithography modeling has been proved to be an adaptable tool in the efforts for the improvement of optical and e-beam lithography over the last years. However since the resolution required in mask making was not so challenging in the past, the main target of EBL simulation tools was the correction of the proximity effect. In this work, simulation results for thin resist layers for a wide range of e-beam energies using a novel electron beam lithography simulator [1] are presented and compared with experimental ones. As it will be proved, the proposed simulator is capable for simulation of dense patterns with critical dimension in the subquartermicron range.

\section{Structure of the simulation tool}

The simulation tool is structured in 4 modules (Figure 1). In the first module the energy deposition $\operatorname{EDF}(r)$ in the resist film due to a "point" beam electron source is calculated [2]. $\operatorname{EDF}(r)$ is the fundamental quantity for EBL simulation since it is independent of the layout to be exposed, the beam shape, dimensions and exposure 
strategy. Thus, the $\operatorname{EDF}(r)$ can be applied in all simulation cases where the beam energy and the composition of the resist and substrate films are the same.

The simulation tool is structured in 4 modules (Figure 1). In the first module the energy deposition $\operatorname{EDF}(r)$ in the resist film due to a "point" beam electron source is calculated [2]. $\operatorname{EDF}(r)$ is the fundamental quantity for EBL simulation since it is independent of the layout to be exposed, the beam shape, dimensions and exposure strategy. Thus, the EDF(r) can be applied in all simulation cases where the beam energy and the composition of the resist and substrate films are the same.

In the second module the beam shape and dimensions are taken into account (Single Pixel Exposure, SEDF(r)) through appropriate convolution of the EDF(r). In the third module the SEDF(r) is convolved with the actual layout $\operatorname{LEDF}(r)$. This module is the most CPU time consuming of the whole simulation procedure, since the energy deposition to a specific point depends strongly on the layout mainly through the contribution of backscattered electrons. The influence range of these electrons depends strongly on the composition of the substrate and the beam energy therefore the CPU time required depends on these parameters. The LEDF(r) is used as input for the simulation of resist development (fourth module) where an appropriate development model and development rate is applied and the final resist profile in two and three dimensions is calculated for the assumed development conditions.

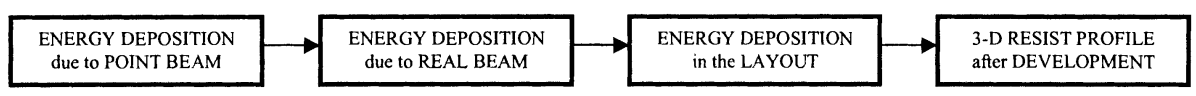

Fig. 1. Process flowchart of EBL simulation tool in the case of conventional resists

\section{Energy deposition calculations}

The energy deposition is the most important quantity for EBL simulation in accordance with the importance of aerial image in the simulation of optical lithography. The method applied in first module of this simulation tool is based on the Boltzmann transport equation and it is well documented in the literature [e.g 12]. This method can be used for the calculation of $\operatorname{EDF}(r)$ in thin resist films on homogeneous substrates (i.e. bulk Si) and on substrates consisting of horizontal layers of different materials (multi layer). Since $\operatorname{EDF}(r)$ is calculated using analytical functions the required CPU time is very small allowing the use of a very fine grid. The CPU time for the calculation of EDF(r) in the case of $0.4 \mu \mathrm{m}$ PMMA on bulk Si using $10 \times 10 \mathrm{~nm}$ grid and $12 \mu \mathrm{m}$ lateral distance at $50 \mathrm{KeV}$ was $26 \mathrm{sec}$ using a Pentium III - 866 MHz computer. The CPU time does not depend on the substrate composition (homogeneous or multi layer); this is not the case for Monte Carlo method where the existence of an interface implies the application of time consuming if -statements.

In figure 2 the $\operatorname{EDF}(\mathrm{r})$ for $20,50,75 \mathrm{keV}$ e-beam energy in the case of $0.4 \mu \mathrm{m}$ PMMA on bulk $\mathrm{Si}$ is presented. From this figure it is obvious that the higher energy causes a more uniform backscattering contribution (larger area) while the contribution from the forward scattered electrons is localized closer to the $Z$ - axis. Due to this behavior the resolution is superior in the case of higher energies while 
the required dose in this cases increases. The $\operatorname{EDF}(\mathrm{r})$ and $\operatorname{SEDF}(\mathrm{r})$ are also valuable for proximity effect correction algorithms. The energy deposition is fitted with a sum of Gaussians. The coefficients and standard deviations of these Gaussians are used as input in the proximity effect correction software.

In figure 3 the effect of resist thickness on the energy deposition is presented. At the bottom of the resist layer the standard deviation of $I_{f}$ (energy deposition due to forward scattered electrons) is significantly larger compared to the according value at the top. Thus for the optimum resolution the combination of thinner resist film higher e-beam energy - best focus beam must be used.

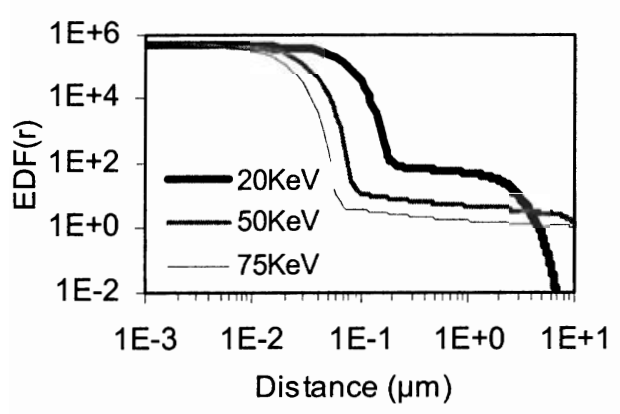

Figure 2: $\operatorname{EDF}(r)$ for various e-beam energies.

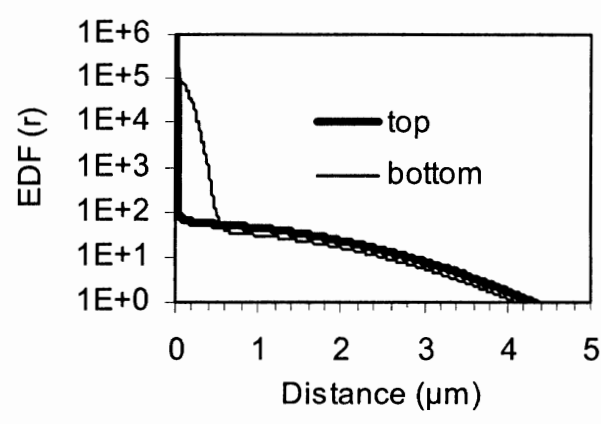

Figure 3: $E D F(r)$ in the case of $0.4 \mu \mathrm{m}$ PMMA on bulk Si for $50 \mathrm{KeV}$ electrons.

\section{Resist Development}

The LEDF(r) is used for the prediction of the resist profile under specific development conditions. The $\operatorname{EDF}(\mathrm{r})$ and consequently $\operatorname{LEDF}(\mathrm{r})$, do not depend strongly on the resist material however the development mechanism of each resist is different and generally it is difficult to develop a simple function that delineates the development mechanism and can be used for the prediction of the resist profile. For instance in some specific resists the developer penetrates the resist film and causes swelling that deteriorates the resolution significantly while in other cases the development time does not affect the resist profile. Therefore for effective simulation a great effort must be given for the development of such a function.

For PMMA a simple function has been developed [3] and used successfully for a long time. In this function the development rate depends on the local energy deposition and on some other parameters that can be measured by experimental techniques. In the present work, these parameters have been calculated using a Dissolution Rate Monitor [4] and have been found different from those mentioned in the literature. More specifically the exponential factor $(\alpha)$ was calculated 4.5 instead of 2.0. In figure 4 the PMMA profile ( $0.8 \mu \mathrm{m}$ thick) of an isolated $0.2 \mu \mathrm{m}$ line is presented for three development times. It is clear that development time of $120 \mathrm{sec}$ is not enough for proper development. On the contrary this development time is enough in the case of $0.4 \mu \mathrm{m}$ PMMA exposed with the same dose.

By using the development rate function mentioned above, extensive simulations have been carried out for the PMMA resist at different resist thicknesses. The layout 
consists of numerous very long lines with nominal linewidth (NLW) of $250 \mathrm{~nm}$ and $150 \mathrm{~nm}$ with spaces $500 \mathrm{~nm}$ and $300 \mathrm{~nm}$ respectively. In figure 5 the simulation and experimental results are compared. The accuracy of simulation in all cases is

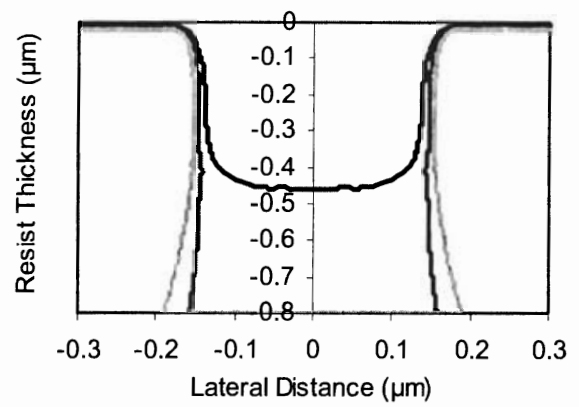

Figure 4: Resist profile for $0.2 \mu \mathrm{m}$ isolated line in $0.8 \mu \mathrm{m}$ thick PMMA. The development times are 120, 180, $240 \mathrm{sec}$

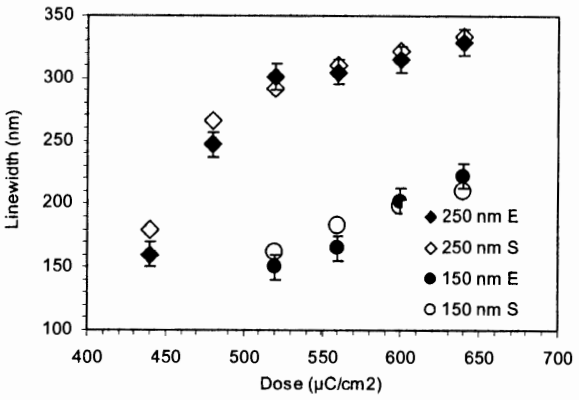

Figure 5: Comparison of experimental width simulation results for $0.4 \mu \mathrm{m}$ PMMA on bulk $\mathrm{Si}(50 \mathrm{KeV})$

satisfactory. The necessary exposures for the calculation of the development rate function and the high resolution patterning were performed by a Leica EBPG-3 ebeam exposure tool operational at 20 and $50 \mathrm{KeV}$. For the metrology a high resolution SEM (LEO 440) was used.

\section{Conclusions}

In the current work a fast electron beam lithography simulator has been applied for the simulation of dense patterns in the $100-250 \mathrm{~nm}$ NLW range at high energies using the conventional positive resist PMMA. Using that simulation tool the effects of beam energy, resist thickness, development time, exposure dose have been studied in depth in order to extract the necessary values for high resolution patterning. In order to improve the simulation's accuracy the development rate parameters have been measured experimentally and found different from those published in the literature. The validity of these values has been verified from the simulation, where the use of literature values produces results far away from the ones observed experimentally.

\section{References}

[1] Glezos N. Raptis I. A fast electron beam lithography simulator based on Boltzmann transport equation IEEE Trans. CAD 1996 15::92-100

[2] Raptis I. Ph.D thesis University of Athens 1996

[3] Neureuther A. Kyser D. Ting C. E-beam resist edge profile simulation IEEE Trans. Electron Devices 1979 26:686-691

[4] Raptis I., Velesiotis D., Vasilopoulou M., Argitis P. Development mechanism study by dissolution monitoring of posistive methacrylate resists Microelec. Eng. 2000 53:489-492 